\title{
An Efficient Synthesis of Bibutenolides from 2,3-Allenoic Acids
}

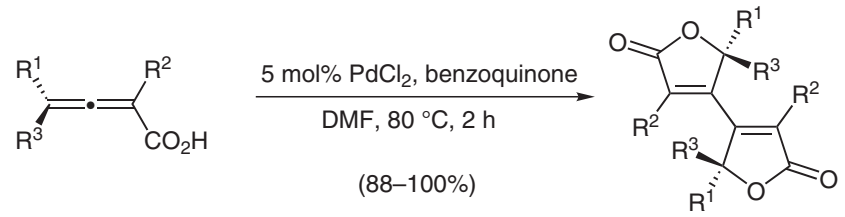

$R^{1}, R^{2}, R^{3}=H$, alkyl, aryl

Significance: A new catalytic system for the preparation of dibutenolides by a cyclization-coupling reaction of 2,3-allenoic acids is described. Three different catalytic systems involving Pd(II) and corresponding additives have been developed, in which the key step is the regeneration of the $\mathrm{Pd}(\mathrm{II})$ species to complete the catalytic cycle. The bicyclization reaction gives excellent yields and diastereoselectivity using optically active 2,3-allenoic acids.
Comment: Allenes possess great potential in organic synthesis as chirality transfer agents (Allenes in Organic Synthesis; H. F. Schuster, G. M. Coppola, Eds.; Wiley: New York, 1984, pp 1-8; S. Ma, Q. Yu J. Org. Chem. 2003, 68, 61496152). Increasing interest in this area includes the use of functionalized allenes to form a variety of cyclic compounds in Pd-catalyzed coupling reactions (S. Ma, H. Xie J. Org. Chem. 2002, 67, 6575-6578). Although naturally occurring dibutenolines are not known, butenolides are common units in many bioactive natural products, including the well know vitamin C. 